\title{
The impact of early palliative care on the quality of care during the last days of life: what does the evidence say?
}

\author{
Sofia C. Zambrano, Monica C. Fliedner, and Steffen Eychmüller
}

\begin{abstract}
Purpose of review
The aim of this review is to critically appraise the existing evidence on 'early palliative care' (EPC), discuss its relationship with advance care planning, and to reflect on the impact of EPC on the quality of care provided during the last days of life.

\section{Recent findings}

There are indicators that EPC may help to avoid aggressive treatment, shorten hospital stay, improve overall quality of life, and to see more frequently dying and death at the preferred place of care.

\section{Summary}

The evidence from randomized controlled trials supports the integration of palliative care early in the disease trajectory. However, in terms of outcomes and quality indicators for care in the last days of life, evidence is still lacking. Predominantly, when it comes to the outcomes which may be more difficult to assess, such as spiritual aspects, or the social network, for which more comprehensive information is needed. These outcomes should not be neglected in palliative care studies, particularly when they can provide meaningful information about patient and family adjustment, and focus on psychosocial aspects rather than physical symptom control.
\end{abstract}

\section{Keywords}

advance care planning, early palliative care, last days of life, palliative care

\section{INTRODUCTION}

Despite a relatively rapid expansion, the provision and implementation of palliative care into clinical care has been hindered by cultural barriers, medical reasoning with an emphasis on 'cure', and societal attitudes towards death and dying. In particular, there exists a general misconception that palliative care is reserved for the last days or weeks of life, a view which is at odds with the emphasis on early integration advocated by the WHO since 1990 [1].

The aim of this review is to critically appraise the existing evidence on 'early palliative care' (EPC), discuss its relationship with advance care planning (ACP), and to reflect on the impact of EPC on the quality of care provided during the last days of life.

\section{WHAT IS EARLY PALLIATIVE CARE?}

There is no accepted definition of EPC applicable to all contexts and clinical situations. EPC or 'early integration of palliative care', in the context of cancer care, is often defined as the 'introduction of specialized palliative care early in the disease trajectory' $[2,3]$. However, the notion of providing early specialist palliative care has been challenged by emphasizing the shortage of palliative care specialists, and the need for EPC to be delivered by oncologists $\left[4^{*}\right]$, geriatricians [5], and general practitioners [5], among many other medical specialists.

Difficulties in understanding and defining EPC are further encountered when determining what 'early' refers to. In a systematic review of peerreviewed articles about early integration of palliative care in oncology, only 18 out of 101 articles

University Center for Palliative Care, Inselspital, University Hospital Bern, Bern, Switzerland

Correspondence to Prof Dr med Steffen Eychmüller, MD, Universitäres Zentrum für Palliative Care, Inselspital, Universitätsspital Bern, Freiburgstrasse 28, SWAN C, CH-3010 Bern, Switzerland.

Tel: +4131632 5107; e-mail: steffen.eychmueller@insel.ch

Curr Opin Support Palliat Care 2016, 10:310-315

DOI:10.1097/SPC.0000000000000240 


\section{KEY POINTS}

- The observed effects of EPC cannot be extrapolated directly to benefits during the last days of life.

- The similarities and differences between EPC and ACP need to be further explored.

- EPC should be 'concurrent' in certain disease pathways, but its initiation should be considered according to the individual needs of the patient.

provided a time frame for the delivery of EPC [6"']. Some studies, including the most recent American Society of Clinical Oncology (ASCO) recommendations, suggest the introduction of EPC at diagnosis of a life-limiting illness [7-9]. Others are more specific and suggest its initiation at diagnosis of advanced illness, or focus on life expectancy, the patient's performance status, or symptom burden $\left[6^{\mathbf{*}}, 10\right]$. The studies suggesting life expectancy propose a wide range of times: from 24 to 12 months or from 6 to 3 months before assumed death $\left[11^{*}, 12,13\right]$. Other authors establish 3 months before expected death as the minimum time frame for palliative care to be considered 'early' [14].

Identifying the appropriate moment in which to address palliative care needs remains a challenge. Not only are illness trajectories often unpredictable, but as mentioned above, most studies implement 'early' at very different moments [15]. An agreement seems to be that palliative care should be integrated before patients experience burdening symptoms for the first time. Therefore, given the prevalence of symptoms and the lack of significant differences in many studies, Gaertner et al. [10] question whether what is considered 'early' could already be too late, if patients are being referred to palliative care when their symptoms are uncontrolled. Bruera et al. describe EPC as an 'integrated care model' in which the oncologist focuses on disease management, while palliative care teams focus on ACP as well as on physical, spiritual, and psychosocial concerns $[10,16,17]$.

\section{Provided by whom?}

The majority of the studies which have implemented EPC approaches have done so through specialist palliative care services. Considering that specialist palliative care services are scarce, general palliative care approaches are needed to incorporate palliative care into the care of the majority of patients with life-limiting illnesses [18"']. Therefore, there is an emphasis on teaching general skills during medical and nursing school and during postgraduate training in the disciplines in which this type of care is needed the most, such as oncology, geriatrics, and general practice, cardiology, and nephrology [18"']. This is particularly relevant because trained specialists can implement an integrated model of palliative care in which they provide basic palliative care on their own but are able to ascertain when they require the support and intervention of specialist palliative care teams [5]. Without this training and experience, it is possible that current practices of late palliative care referrals continue to occur.

\section{Differences and similarities between early palliative care and advance care planning}

The primary purpose of ACP is to ensure that the care provided to patients meets their preferences by respecting their decisions and autonomy [19"]. Although ACP can be regarded as a long-lasting process and involves more comprehensive interventions than just defining advance directives, EPC seems to have a wider approach focused on minimizing or dealing with the illness and the effects of its treatment. Yoong et al. [20] proposed seven key elements of EPC in cancer care: rapport building, symptom management, addressing coping strategies, establishing illness understanding, discussing treatments, end-of-life planning, and engaging family members. These key aspects cannot easily be differentiated from those of complex ACP approaches [21] and therefore, even though they are generally described as different entities, it is still difficult to differentiate the concepts of ACP and EPC.

\section{What aspects and from whose perspective?}

Palliative care clinicians establish a relationship first and determine patient needs and preferences for information before initiating the discussion of end-of-life planning, when the health status of the patient has changed [20]. However, there seems to be a lack of clarity on the content and 'effective component' of EPC interventions [22]. From the patients' perspective, the literature defines certain topics and domains that need to be addressed in a comprehensive early assessment and planning. For example, symptom management, preparation for death, achieving a sense of completion, decisions about treatment preferences, and being treated as a 'whole person' are generally regarded by stakeholders as goals of treatment [23]. However, goals which are important for healthcare professionals may not be as meaningful to patients: A study found that patients gave more relevance than physicians to 
being mentally aware, having funeral arrangements planned, not being a burden, helping others, and coming to peace with God [23].

\section{IMPACT ON THE LAST DAYS OF LIFE}

A landmark randomized controlled trial (RCT) about the impact of EPC found that patients experienced improved quality of life, received less aggressive care, were more likely to establish advance directives, and lived longer [9]. Other studies identified improvements in symptoms [3,24], depression $[24,25]$, distress [26], quality of life [3,24,27], and satisfaction with care and communication $[3,28,29]$. Family members reported emotional and spiritual growth and were more satisfied with care feeling that the needs of their relative were addressed appropriately [30,31]. Palliative care interventions also influence health service utilization: Patients are less likely to be admitted to the hospital, ICU, or emergency department $[29,32]$, and have an increased likelihood of dying in their preferred place $[29,33]$. Despite highlighting that some result discrepancies between RCTs may be related to differences in the nature and type of interventions, the timing of initiation or EPC, as well as defined outcomes, several systematic reviews of EPC further confirm its positive effects $\left[6^{\boldsymbol{*}}, 15,34^{*}, 35^{-"}\right]$. Most importantly, the observed effects cannot be extrapolated directly to benefits during the last days of life, because the majority of the studies do not measure the outcomes which are more relevant to the last days of life, except when measuring place of death and certain measures of healthcare utilization.

The OPCARE 9 collaboration sought consensus about quality indicators, including care outcomes relevant to the last days of life [36,37]. Among the highest ranking parameters were managing physical symptoms such as pain, nausea/vomiting, dyspnoea, and other aspects of care such as anxiety, and timely communication about approaching death [36]. In the proposed list of topics by Raijmakers et al. [37] were the availability of a family room, a home visit after the patient's death, preferred place of death, less patients receiving chemotherapy, limited need for pain control, fewer gastrointestinal symptoms, and adequate communication between professionals and the patient and families, as well as the use of care plans, pathways or guidelines.

The evidence from RCTs supports the integration of palliative care early in the disease trajectory. However, in terms of the proposed outcomes and quality indicators for care in the last days of life, evidence is still lacking. Predominantly, when it comes to the outcomes which may be more difficult to assess, such as spiritual aspects, or for which more comprehensive information is needed, such as the social network. These outcomes should not be neglected in palliative care studies, particularly when they can provide meaningful information about patient and family adjustment and focus on psychosocial aspects rather than physical symptom control $\left[38^{-"}, 39\right]$.

More specific aspects which can be considered as part of better care during the last days of life can be more easily identified in ACP studies than in EPC studies, in which matched preferences for place of death with actual place of death tend to be found after most interventions, as well as less use of resuscitation and life-sustaining equipment [21]. This may be the case because ACP studies include these end-of-life outcomes, whereas the majority of EPC studies focus on more general health care and economic outcomes.

Cost-saving studies of palliative care, in which invasive and expensive treatments are reduced, can provide some evidence that patients receive less aggressive treatments at the end-of-life [40,41]. Studies on personal costs such as out of pocket costs for family members of patients in palliative care are still scarce [42].

Of the many studies identified, only one assessed the effects of EPC on the treatment during the last week of life. Zhang et al. [43] found that patients who had discussions about the end-of-life about 6 months before death, including discussions about treatment preferences, were more likely to choose more conservative treatments and to be more realistic about their prognosis. In their study, an end-of-life conversation was associated with a good quality of life in the last week of life in terms of being less likely to die in the ICU, to be resuscitated, having less physical distress, and it was also associated with $35.7 \%$ less hospital costs during the last week of life. They did not find differences in psychological distress or survival time. Further analyses showed that higher medical costs were associated with more distress and worse overall quality of death from the caregiver's perspective $[43,44]$.

\section{CLINICAL AND RESEARCH IMPLICATIONS}

Reviewing the effect of 'EPC' on the quality of death and dying has led us to reflect on three main aspects: what are the differences between EPC and ACP?, which are the essential factors that may contribute to a 'good death'?, and what are the meaningful outcomes of this relatively new approach called 'EPC'? The answers to these questions encompass a more profound discussion on 'what is the effective component of palliative care?', and even further, on 'what is palliative care', 'EPC', and 'ACP'? 
In good clinical practice, early and comprehensive assessment and management of potential stressful situations in deteriorating health conditions for patients, family carers but also for health professionals is warranted. In this context, timing ('right time for the right patient') might be an important issue.

\section{What are the differences between early palliative care and advance care planning?}

'Early' in the clinical setting is a poorly defined term. From our perspective, 'concurrent' may be a better, and less bothersome term. As Bruera and Hui [16] pointed out palliative care or EPC is not a matter of timing but of content. Patients may fear 'early' palliative care, as may do specialists and health insurance companies (see reimbursement policy in the United States for hospice care). In its implementation, 'palliative care' has created despair and new fears (e.g., $\left[45^{-*}, 46\right]$ ), and even after decades of routine implementation in many countries and settings, palliative care is still often associated with dying and death $\left[47^{\boldsymbol{*}}, 48\right]$. Based on the literature, a new 'branding' of what we do and offer is important: 'EPC' is in other words 'concurrent $\mathrm{ACP}^{\prime}$ in chronic progressive conditions including the last months, weeks, and days of life, and explicitly addressing fears in terms of 'dying and death'. By following this interpretation, EPC would add pragmatic planning driven by the patients' and families' agenda and consequently, would structure the questions (patient-related meaningful outcomes) that should be addressed, offering a clear process based on a high level of compassionate communication.

\section{Which are the essential factors that may contribute to a 'good death'?}

As mentioned above beyond the question of 'when', the 'what' is crucial: evidence shows that palliative care adds a problem-based, patient-centred, interprofessional approach to assessment and care planning, which complements the pure medical diagnosis-based reasoning prevailing in the medical context.

End-of-life discussions and evaluating the goals early enough to avoid emergency situations and crisis decisions are specifically helpful to improve quality of care, quality of life, and the appropriateness of medical interventions (e.g., $[3,43,49])$. In addition, certain barriers need to be overcome to facilitate working hand-in-hand between specialists, the palliative care team and/or primary care clinicians $[50,51]$.
There is no research directly connecting EPC interventions and quality of death in an experimental design. There are indicators that EPC may help to avoid aggressive treatment, shorten hospital stay, improve overall quality of life, and possibly to see more frequently dying and death at the preferred place of care. However, taking into account the character of mixed and complex interventions with various potentially confounding factors, and the heterogeneity of involved populations and health systems (which may influence endpoints such as place of care), it is still unknown whether there is any causality between EPC interventions and 'a good dying and death'.

\section{What are the meaningful outcomes of early palliative care?}

Questions regarding patient priorities as to which outcomes of care are more relevant in the last days of life remain unexplored [52]. How can services best identify and meet patients' individual needs, and how can they effectively measure them? Research with a more narrow focus on the late stage of life is required [53]. During the last days of life, the symptoms of dying as well as the illness itself and the awareness of dying may affect the patient at a level in which it had not before, and many areas of patient and family need could be neglected, or given less importance based on current outcome standards [39].

There is no agreed upon core outcome set for best care for the dying or indicators of a 'good death'. Much more work is needed to better understand common and individual outcomes from the perspective of patients (if possible to be captured) and family caregivers. Partnership research projects bringing together all stakeholders, including health professionals, patients, and families, may provide such information in the future.

\section{CONCLUSION}

The issue of identifying an appropriate time for 'early' palliative care requires a reconsideration of the characteristics of the specific setting, considering that although some patients may have a longer life expectancy as in breast cancer or congestive heart failure, in other diagnoses the model of EPC delivery should include specialized palliative care teams in addition to the basic palliative care provided by superficially trained professionals [17].

EPC and ACP are interrelated concepts which need to be more clearly defined. We suggest that complex ACP interventions provide comprehensive palliative care irrespective of a disease time frame, 
whereas EPC focuses on the clinical effects of disease early on. The components of what makes a difference for the patients and families via EPC or ACP need to be incorporated into routine clinical care for patients with a life-limiting illness.

\section{Acknowledgements}

None.

\section{Financial support and sponsorship}

In the writing of the manuscript the authors received no financial support and/or sponsorship.

\section{Conflicts of interest}

There are no conflicts of interest.

\section{REFERENCES AND RECOMMENDED READING}

Papers of particular interest, published within the annual period of review, have been highlighted as:

- of special interest

- of outstanding interest

1. World Health Organisation. Cancer pain relief and palliative care. WHO expert committee on cancer pain relief and active supportive care. Geneva: World Health Organisation; 1990.

2. Gaertner J, Weingartner V, Wolf J, Voltz R. Early palliative care for patients with advanced cancer: how to make it work? Curr Opin Oncol 2013; 25:342-352.

3. Zimmermann C, Swami N, Krzyzanowska M, et al. Early palliative care for patients with advanced cancer: a cluster-randomised controlled trial. Lancet 2014; 383:1721-1730.

4. Hui $D$, Finlay $E$, Buss $M K$, et al. Palliative oncologists: specialists in the

- science and art of patient care. J Clin Oncol 2015; 33:2314-2317.

Interesting piece highlighting what palliative oncology is and the role of palliative

oncologists in bridging oncology and specialist palliative care.

5. Quill TE, Abernethy AP. Generalist plus specialist palliative care - creating a more sustainable model. N Engl J Med 2013; 368:1173-1175.

6. Hui D, Kim YJ, Park JC, et al. Integration of oncology and palliative care: a

- systematic review. Oncologist 2015; 20:77-83.

This systematic review identified 101 articles which point to different aspects (clinical, administrative, educational and research) of early integration of palliative care into oncology.

7. Ferris FD, Bruera E, Cherny N. Palliative cancer care a decade later: accomplishments, the need, next steps: from the American Society of Clinical Oncology. J Clin Oncol 2009; 27:3052-3058.

8. Smith TJ, Temin S, Alesi ER. American Society of Clinical Oncology provisional clinical opinion: the integration of palliative care into standard oncology care. J Clin Oncol 2012; 30:880-887.

9. Temel JS, Greer JA, Muzikansky A, et al. Early palliative care for patients with metastatic non-small-cell lung cancer. N Engl J Med 2010; 363:733-742.

10. Gaertner J, Wolf J, Frechen S, et al. Recommending early integration of palliative care - does it work? Support Care Cancer 2012; 20:507-513.

11. Amano $\mathrm{K}$, Morita $T$, Tatara $R$, et al. Association between early palliative care

- referrals, inpatient hospice utilization, and aggressiveness of care at the end of life. J Palliat Med 2015; 18:270-273.

This retrospective study of 266 cancer decedents in Japan found that early palliative care referrals facilitated hospice admission and less aggressive end of life care.

12. Nieder $C$, Tollali $T$, Haukland $E$, et al. Impact of early palliative interventions on the outcomes of care for patients with nonsmall cell lung cancer. Support Care Cancer 2016. [Epub ahead of print]

13. Hui $\mathrm{D}, \mathrm{Kim} \mathrm{SH}$, Roquemore J, et al. Impact of timing and setting of palliative care referral on quality of end-of-life care in cancer patients. Cancer 2014; 120:1743-1749.

14. Davis MP, Carrino $C$. Promoting further use of palliative care in cancer care centers. Expert Rev Qual Life Cancer Care 2016; 1:213-220.

15. Dalgaard KM, Bergenholtz $H$, Nielsen ME, Timm $H$. Early integration of palliative care in hospitals: a systematic review on methods, barriers, and outcome. Palliat Support Care 2014; 12:495-513.

16. Bruera $E$, Hui $D$. Integrating supportive and palliative care in the trajectory of cancer: establishing goals and models of care. J Clin Oncol 2010; 28:40134017.
17. Greer JA, Jackson VA, Meier DE, Temel JS. Early integration of palliative care services with standard oncology care for patients with advanced cancer. CA Cancer J Clin 2013; 63:349-363.

18. Gaertner J, Maier BO, Radbruch L. Resource allocation issues concerning n. early palliative care. Ann Palliat Med 2015; 4:156-161.

This review article discusses early palliative care provided through general and specialist approaches and concludes that both approaches must coexist as a joint effort.

19. Rietjens J, Korfage I, van der Heide A. Advance care planning: not a panacea. - Palliat Med 2016; 30:421-422.

This editorial discusses the advantages, challenges, dilemmas and pitfalls of engaging patients in advance care planning (ACP) and suggests a way forward for research studies to provide more meaningful evidence on the usefulness of ACP.

20. Yoong J, Park ER, Greer JA. Early palliative care in advanced lung cancer: a qualitative study. JAMA Intern Med 2013; 173:283-290.

21. Brinkman-Stoppelenburg A, Rietjens JA, Heide A. The effects of advance care planning on end-of-life care: a systematic review. Palliat Med 2014; 28:1000-1025.

22. Block SD, Billings JA. A need for scalable outpatient palliative care interventions. Lancet 2014; 383:1699-1700.

23. Steinhauser KE, Christakis NA, Clipp EC, et al. Factors considered important at the end of life by patients, family, physicians, and other care providers. JAMA 2000; 284:2476-2482.

24. Bakitas M, Lyons KD, Hegel MT. Effects of a palliative care intervention on clinical outcomes in patients with advanced cancer: the project ENABLE II randomized controlled trial. JAMA 2009; 302:741-749.

25. Pirl WF, Greer JA, Traeger L, et al. Depression and survival in metastatic nonsmall-cell lung cancer: effects of early palliative care. J Clin Oncol 2012; 30:1310-1315.

26. Aiken LS, Butner J, Lockhart CA, et al. Outcome evaluation of a randomized trial of the PhoenixCare intervention: program of case management and coordinated care for the seriously chronically ill. J Palliat Med 2006; 9:111-126.

27. Clark MM, Rummans TA, Sloan JA, et al. Quality of life of caregivers of patients with advanced-stage cancer. Am J Hosp Palliat Care 2006; 23:185-191.

28. Engelhardt JB, McClive-Reed KP, Toseland RW, et al. Effects of a program for coordinated care of advanced illness on patients, surrogates, and healthcare costs: a randomized trial. Am J Manag Care 2006; 12:93-100.

29. Brumley $R$, Enguidanos $S$, Jamison $P$, et al. Increased satisfaction with care and lower costs: results of a randomized trial of in-home palliative care. J Am Geriatr Soc 2007; 55:993-1000.

30. Roza KA, Lee EJ, Meier DE, Goldstein NE. A survey of bereaved family members to assess quality of care on a palliative care unit. J Palliat Med 2015; 18:358-365.

31. Hales $S$, Chiu A, Husain A, et al. The quality of dying and death in cancer and its relationship to palliative care and place of death. J Pain Symptom Manage 2014; 48:839-851.

32. Khandelwal N, Benkeser DC, Coe NB, Curtis JR. Potential influence of advance care planning and palliative care consultation on ICU costs for patients with chronic and serious illness. Crit Care Med 2016; 44:14741481; Published ahead of time DOI: 10.1097/CCM.0000000000001675.

33. Abel J, Pring A, Rich A, et al. The impact of advance care planning of place of death, a hospice retrospective cohort study. BMJ Support Palliat Care 2013; 3:168-173.

34. Tassinari D, Drudi F, Monterubbianesi MC, et al. Early palliative care in - advanced oncologic and nononcologic chronic diseases: a systematic review of literature. Rev Recent Clin Trials 2016; 11:63-71.

This is the most recent systematic review that combines evidence for early palliative care (EPC) in malignant and nonmalignant illnesses.

35. Davis MP, Temel JS, Balboni T, Glare P. A review of the trials which examine

- early integration of outpatient and home palliative care for patients with serious illnesses. Ann Palliat Med 2015; 4:99-121.

While identifying evidence in favour of EPC in the outpatient setting, the authors provide critical arguments on why better research is needed in this area and how it can be improved.

36. Eychmueller S, Grossenbacher-Gschwend B, Montag T, Voltz R. What we should achieve: core outcome sets for care in the dying phase. In: Ninth world research congress of the European Association for Palliative Care; June 9-11, 2016, Dublin: Palliative Medicine; 2016.NP1-NP401.

37. Raijmakers $N$, Galushko $M$, Domeisen $F$, et al. Quality indicators for care of cancer patients in their last days of life: literature update and experts' evaluation. J Palliat Med 2012; 15:308-316.

38. Michael N, O'Callaghan $\mathrm{C}$, Brooker JE, et al. Introducing a model incorporat-

- ing early integration of specialist palliative care: a qualitative research study of staff's perspectives. Palliat Med 2016; 30:303-312.

This qualitative study explored staff perceptions of the challenges associated with providing EPC and discuss its implications for services implementing new models of palliative care delivery.

39. Currow DC. The PRISMA symposium 3: lessons from beyond Europe. Why invest in research and service development in palliative care? An Australian perspective. J Pain Symptom Manage 2011; 42:505-510. 
40. Smith S, Brick A, O'Hara S, Normand C. Evidence on the cost and costeffectiveness of palliative care: a literature review. Palliat Med 2014; 28:130150.

41. Gade $G$, Venohr I, Conner D, et al. Impact of an inpatient palliative care team: a randomized control trial. J Palliat Med 2008; 11:180-190.

42. Penders YW, Rietjens J, Albers G, et al. Differences in out-of-pocket costs of healthcare in the last year of life of older people in 13 European countries. Palliat Med 2016. [Epub ahead of print]

43. Zhang $B$, Wright $A A$, Huskamp $H A$, et al. Healthcare costs in the last week of life: associations with end-of-life conversations. Arch Intern Med 2009; 169:480-488.

44. Zhang B, Nilsson ME, Prigerson HG. Factors important to patients' quality of life at the end of life. Arch Intern Med 2012; 172:1133-1142.

45. Zimmermann C, Swami N, Krzyzanowska M, et al. Perceptions of palliative

- care among patients with advanced cancer and their caregivers. Can Med Assoc J 2016; 188:E217-E227. [Epub ahead of print]

This qualitative follow-up study investigated patients' and caregivers' perceptions of palliative care after participating in a randomized controlled trial of EPC. The authors found that both groups (intervention and nonintervention) had similar negative perceptions of palliative care. After completion of the study, those in the intervention group had a wider definition of palliative care but still held a negative perception of the term.
46. Maciasz RM, Arnold RM, Chu E, et al. Does it matter what you call it? A randomized trial of language used to describe palliative care services. Support Care Cancer 2013; 21:3411-3419.

47. Weil J, Weiland TJ, Lane $\mathrm{H}$, et al. What's in a name? A qualitative exploration of

- what is understood by 'palliative care' in the emergency department. Palliat Med 2015; 29:293-301. [Epub ahead of print]

Staff perceptions of palliative care were investigated in this qualitative study. The authors highlight several contradictions in staff's understanding of palliative care and discuss its implications on palliative care referrals.

48. Gott M, Seymour J, Ingleton C, et al. 'That's part of everybody's job': the perspectives of healthcare staff in England and New Zealand on the meaning and remit of palliative care. Palliat Med 2012; 26:232-241.

49. Holmes HM, Hayley DC, Alexander GC, Sachs GA. Reconsidering medication appropriateness for patients late in life. Arch Intern Med 2006; 166:605-609.

50. Kelley AS, Meier DE. Palliative care - a shifting paradigm. N Engl J Med 2010; 363:781-782

51. Alesi ER, Fletcher DS. Integrating palliative care into oncology care: confronting the barriers. Oncology (Williston Park) 2013; 27 :.

52. Chan R, Webster J. End-of-life care pathways for improving outcomes in caring for the dying. Cochrane Database Syst Rev 2010; Cd008006.

53. Costantini M, Lunder U. OPCARE 9 - a European perspective on the last days of life. Eur J Palliat Care 2012; 4:175 